\title{
Phenotyping of Tomato for SolCAP and Onward into the Void
}

\author{
John W. Scott \\ Gulf Coast Research and Education Center, IFAS, University of Florida, 14625 CR 672, Wimauma, FL 33598
}

Additional index words. fruit shape, fruit color, fruit disorder, flavor, Lycopersicon esculentum, Solanum lycopersicon

\begin{abstract}
Standardized phenotyping is being used in tomato (Solanum lycopersicum) and potato (S. tuberosum) as part of the USDA-funded Solanaceae coordinated agricultural project (SolCAP). In tomato, a panel of 480 lines, 144 fresh market, 144 processing, 44 vintage lines, 48 landraces, and 101 related Solanum species accessions, are being grown over two seasons at multiple locations. These tomato genotypes will also be screened for over 7600 single nucleotide polymorphism markers that are being developed. Characters being phenotyped are hypocotyl color, plant habit, inflorescence (simple or compound), flower fasciation, flowering time, time to maturity, size of blossom scar, and pedicel type (jointless or jointed). Transverse and equatorial cross-sections of fruit are also being scanned to obtain measures of fruit shape and color by tomato analyzer computer software. Other fruit characters being measured are $\mathrm{pH}$, titratable acidity, and soluble solids. Some useful information may come from this phenotyping and genotyping effort. However, in this author's opinion, the value of measuring some of the traits is very limited in the face of much more important breeding traits that are difficult to fit into a standardized phenotyping format. For instance, proper assessment of several fruit disorders is not being attempted, but such data might yield some invaluable marker information. Part of this is the result of funding limitations for the phenotyping effort. However, a major benefit of the SolCAP analysis will be the development of markers that will differentiate the lines being studied, because there is a present lack of polymorphisms in tomato germplasm where wild species introgressions are minimal or distant in time. This will allow breeders to proactively select for recurrent parent backgrounds in backcrossing projects and may aid in the identification of associations of markers with important characteristics. As one looks to the future of standardized phenotyping, there are some important traits that will be difficult to phenotype such as tomato flavor, which is quite subjective and for which there are no good objective measures.
\end{abstract}

Tomato is one of the major model crops for genetic analysis and it has been widely studied (Suzuki et al., 2009). It is also an important commercial crop for both fresh market and processing industries as well as a popular home garden vegetable for the seed packet trade. Thus, considerable breeding has taken place since the late 1800 s and especially since the 1930 s. A well-recognized constraint to present-day breeding efforts has been the narrow gene base and subsequent lack of polymorphisms, except in cases in which the breeding material has introgressions from wild species for disease resistance or other genes (Miller and Tanksley, 1990; Williams and St. Clair, 1993). Genomic databases are being developed at increasingly rapid rates, and in 2009, the first version of the shot gun tomato sequence became available at the Sol Genomics Network (SGN) web site (http://solgenomics.net/index.pl). For genomic data to better lead to crop improvement, it is important that translational genomics be part of the process. The USDA AFRI Coordinated Agricultural Projects (CAPs) are an effort to address this. Tomato/solanaceae issues regarding translational genomics were discussed by Francis (2005) and later the SolCAP project was funded in 2008 with a focus on potato and tomato. Standardized phenotyping originated with the mouse community (Abbott, 1999) to allow researchers to effectively compare mouse strains used as models for human diseases. Standardized phenotyping is now being applied to various plant species, espe-

Received for publication 9 Mar. 2010. Accepted for publication 12 Apr. 2010.

e-mail e-mail jwsc@ufl.edu. cially tree fruits (Peace and Norelli, 2009), largely in response to the development of genomic information. The objectives of this report are: 1) to provide a brief introduction to the SolCAP project with a focus on tomato; and 2) to discuss the pros and cons about the tomato standardized phenotyping that is being done for the project. Additional viewpoints as to standardized phenotyping are also presented.

\section{SolCAP}

The overriding premise of SolCAP (http://solcap.msu.edu/) is that there is a major gap between genomic information and breeding. In the project, an important trait to be studied, common between tomato and potato, is carbohydrate and sugar metabolism. The germplasm used is elite and breeder-driven. Genotyping will be done after developing single nucleotide polymorphisms (SNPs) at a University of California, Davis laboratory. Besides the germplasm being used for standardized phenotyping, there will be flexible funding to genotype additional populations for the potato and tomato communities for traits of interest and as needs emerge. Database mining and management at SGN and SolCAP web sites will be facilitated. The extension component will focus on plant breeder education using PBGworks at eXtension (http://pbgworks.hort.oregonstate. edu/) with the goal of partnering with other CAPs. Education will consist of curriculum development, breeder workshops, and development of a graduate course. The five broad research objectives of the project are: to collect standardized phenotypic data across multiple environments for tomato and potato; to develop extensive sequence data of expressed genes and identify 7600 SNP markers, some associated with candidate genes for carbohydrate or vitamin biosynthetic pathways; to establish centralized facilities for SNP genotyping of germplasm panels in tomato and potato; to address regional, individual program and emerging needs through a small grants program; and to create integrated, breeder-focused resources for genotypic and phenotypic analysis by leveraging existing databases and resources at SGN.

\section{STANDARDIZED PHENOTYPING OF TOMATO}

The plan is to test 480 lines, including 144 fresh market, 144 processing, 44 vintage lines, 47 landraces, and 101 wild species accessions. The processing lines will be trialed in California by Campbell's and at the Ohio State University in Wooster. The fresh market lines will be tested at the University of California, Davis, North Carolina State University in Mills River, and at the University of Florida in Wimauma. Vintage lines will be trialed at North Carolina State, Ohio State, and at Cornell University in Ithaca, NY. Two trials will be conducted at each location. Spreadsheets have been distributed for data entry where only possible data can be successfully entered. For instance, if one is to enter a 0 for absence or a 1 for presence of a character but the letter "o" is mistakenly entered, the spreadsheet will reject the entry and it will have to be redone. For each location, passport information will be recorded to describe the location and conditions of the trial (Table 1). The standardized phenotypic characters being recorded are listed in Table 2. Of the traits being measured, one of the key features is the use of tomato analyzer software to measure fruit shape (Brewer et al., 2006) and interior 
Table 1. Required field passport data for tomato standardized phenotype trials.

\begin{tabular}{l}
\hline I. Location \\
Degrees and minutes of $\mathrm{N}$ (north) or $\mathrm{S}$ \\
$\quad$ (south) latitude \\
Degrees and minutes of $\mathrm{E}$ (east) or $\mathrm{W}$ \\
$\quad$ (west) longitude \\
Elevation (meters above sea level) \\
Name of farm or institute \\
Street address \\
Year (YYYY) \\
Sowing date $(\mathrm{MM} / \mathrm{DD} / \mathrm{YY})$ \\
Planting date $(\mathrm{MM} / \mathrm{DD} / \mathrm{YY})$ \\
Transplanting date $(\mathrm{MM} / \mathrm{DD} / \mathrm{YY})$ \\
First harvest date $(\mathrm{MM} / \mathrm{DD} / \mathrm{YY})$ \\
Last harvest date $(\mathrm{MM} / \mathrm{DD} / \mathrm{YY})$ \\
\\
II. Seasonal \\
Rainfall \\
Irrigation \\
Soil description \\
Texture \\
Fertilization practices \\
Herbicides used \\
Disease management \\
Insect management \\
\hline
\end{tabular}

Table 2. Standardized phenotype descriptions for tomato SolCAP project.

\begin{tabular}{l}
\hline I. Independent variables \\
Replicate \\
Line \\
Plant
\end{tabular}

II. Dependent variables: plant

Seedling - anthocyanin present or absent

Plant habit-determinate or indeterminate

Inflorescence-single, intermediate, compound

Flower: fasciation present

Pedicel_-jointed or jointless

Time of anthesis - third truss

Time of maturity - third set

Fruit blossom scar-size

Fruit-shape with tomato analyzer

Fruit-interior color with tomato analyzer

III. Dependent variables: tomato fruit quality Soluble solids-Brix

$\mathrm{pH}$

Titratable acidity

color (Darrigues et al., 2008). The plan is to scan equatorial and cross-sections of 10 fruit per plot on flatbed scanners and ship the images to Ohio where the images will be processed with the computer software.

\section{PHENOTYPING LIMITATIONS}

Analysis of the phenotypic and genotypic data will likely result in some interesting and useful discoveries. How the standardized phenotyping translates to crop improvement remains to be seen. Probably the limiting factor in the use of genomic information is obtaining accurate phenotypic data. Several of the traits to be measured such as anthocyanless hypocotyls (a, $a a, a h$ ), determinate plant habit $(s p)$, compound inflorescence $(s)$, fasciated flowers/fruit $(f)$, and jointless pedicel $(j-2)$ have been located on the classical chromosome map published over 20 years ago (Mutchler et al., 1987). Improved mark- ers and more precise map locations for these traits may result from this project. However, having improved markers for these characters is not likely going to be of critical importance for tomato improvement. Anthocyanin in hypocotyls is easy to score in seedlings without markers. Markers already exist for $s p$ and $j-2$. In large-fruited fresh market tomatoes, it is not so critical to have a marker for jointless; the difficulty is to develop jointless tomatoes that are as good as existing jointed tomatoes because defects such as rough blossom scars, off shapes, and fruit cracking are often worse in jointless tomatoes under many growing conditions. Breeding fresh market tomato cultivars that maintain a smooth, symmetrical, round shape with smooth blossom scars is of critical importance. Much information will be obtained for fruit shape, but it may not lead to the association of markers with reliable good shape because of the sampling procedure. Although there are multiple locations and seasons, the small numbers of fruit sampled (which cannot be helped as a result of budget and time constraints) may not be adequate to identify lines that are not prone to off-shaped fruit formation. The same can be said for measuring blossom scar size, an important trait where cultivars need to be smooth (Barten et al., 1993). Some cultivars will get rough scars under some conditions, but if the fruit are not sampled under those conditions, the differences will not be in the data set. For example, in a given trial perhaps almost all early fruit would be smooth, but after some cold weather, fruit midway up the plant on some lines would be rough, whereas fruit of others retain their smoothness. If in our trials the early fruit are sampled, the differences are not seen nor analyzed.

Otherwise, many important traits are not being scored. Tomato fruit disorders are often difficult to select against because they are not always expressed. Graywall (Stall, 1991) can be used as an example. In a breeding program, if early-generation selection is done with no graywall pressure, then many superior selections can be made that, in a future season, are found to be susceptible to graywall and must therefore be discarded. Unfortunately, this occurs after considerable time and effort have been expended on this material. In the standardized phenotyping trials, if there was graywall pressure at any of the locations, it would be a good opportunity to categorize the lines for this problem. The identification of a SNP(s) associated with resistance could be of monumental importance because it could lead to the incorporation of resistance into all future cultivars. However, because graywall is not on the list of traits to be measured (Table 2), this opportunity to find the marker by association mapping could be missed. Researchers doing the trials can do optional ratings for traits such as graywall and thus all might not be lost. The point is there are constraints on the standardized phenotyping, which is underfunded, a fact readily admitted by the project leaders. This discussion is not meant to be critical of the project, but rather to point out that it is difficult to do standardized phenotyping on the myriad of important traits that are really needed in a breeding program. Obtaining good phenotypic data takes time, and those doing the phenotyping may not have time to obtain all the data that might be desired. Some traits are fairly straightforward to measure, whereas others are not so amenable to standardized phenotyping. Flavor is an important trait that fits the latter category. Consumers have found considerable dissatisfaction with the flavor of tomatoes in the marketplace (Bruhn et al., 1991; Hobson and Bedford, 1989). Breeding for flavor is quite complicated as a result of the quantitative genetic control of sugars, acids, and $\approx 16$ aromatic volatiles where environmental influences are profound (Scott, 2002). It would be difficult to assess flavor by standardized phenotyping simply because of differences in flavor preferences among the persons doing the tasting at each location. Another problem would be taste fatigue during the sampling process. The sugars, acids, and volatiles could be measured to provide some objective data, but as a result of environmental effects, sampling procedures might well result in unwanted variation even when extreme care is taken. In short, traits like tomato flavor are not easy to breed for without selectable markers. However, developing useful markers cannot be done without proper phenotyping. On the other hand, if all the people conducting the trials decided a particular line or two had outstanding flavor, progress to find useful markers might be made. Hopefully, the outstanding flavor line(s) would not also have undesirable characteristics such as low yield or small fruit size (Georgelis et al., 2006).

I feel one of the best things that will come from the lines being phenotyped and genotyped will be the discovery of polymorphisms between lines in the breeding programs. If the polymorphic SNPs are reasonably scattered throughout the genome, one could then do proactive selection for background genes in a backcrossing program and thus save valuable time in cultivar development. Additionally, having genotypic data in hand will aid tremendously both in selecting parents for genetic studies and in identifying candidate regions in which genes of interest may be located. Returning to the graywall example, if a breeder knows which of their entries are resistant and susceptible to graywall, the polymorphic markers between the groups could be targets for validation work to locate graywall-linked markers as opposed to looking for markers from scratch. Furthermore, some of the entries from Florida contain genes for resistance to tomato yellow leaf curl virus, although we have not yet located all of the resistance genes ( $\mathrm{Ji}$ et al., 2009). SNPs polymorphic between these lines and all the susceptible lines would be candidates for linkage to resistance genes, providing likely targets for confirmation studies. An outstanding feature of the SolCAP plan is to have a small grants program to address regional, individual program and emerging 
needs within the solanaceae community. The markers being developed can be used for quantitative trait locus validation studies on phenotyped populations of the grantees. The SolCAP project is off to a good start and will no doubt be of great benefit to the tomato and potato breeding communities. For tomato, the standardized phenotyping may result in some useful discoveries but appears to have some shortcomings. However, it is a start, and it provides something to build on for the future.

\section{Literature Cited}

Abbott, A. 1999. Database to standardize mouse phenotyping. Nature 401:833.

Barten, J.H.M., Y. Elkind, J.W. Scott, S. Vidavski, and N. Kedar. 1993. Diallel analysis over two environments for blossom-end scar size in tomato. Euphytica 65:229-237.

Brewer, M.T., L. Lang, K. Fujimura, N. Dujmovic, S. Gray, and E. van der Knaap. 2006. Development of a controlled vocabulary and software application to analyze fruit shape variation in tomato and other species. Plant Physiol. 141: $15-25$
Bruhn, C.M., N. Feldman, C. Garlitz, J. Harwood, E. Ivans, M. Marshall, A. Riley, D. Thurber, and E. Williamson. 1991. Consumer perceptions of quality: Apricots, cantaloupes, peaches, pears, strawberries, and tomatoes. J. Food Qual. 14:187-195.

Darrigues, A., J. Hall, E. van der Knaap, D.M. Francis, N. Dujmovic, and S. Gray. 2008. Tomato analyzer-color test: A new tool for efficient digital phenotyping. J. Amer. Soc. Hort. Sci. 133:579-586.

Francis, D. 2005. Translational genomics and the Solanaceae. Rept. Tomato Genet. Coop. 55:912.

Georgelis, N., J.W. Scott, and E.A. Baldwin. 2006. Inheritance of high sugars from tomato accession PI270248 and environmental variation between seasons. J. Amer. Soc. Hort. Sci. 131: $41-45$.

Hobson, G.E. and L. Bedford. 1989. The composition of cherry tomatoes and its relation to consumer acceptability. J. Hort. Sci. 64:321329.

Ji, Y., J.W. Scott, D.J. Schuster, and D.P. Maxwell. 2009. Molecular mapping of $T y-4$, a new tomato yellow leaf curl virus resistance locus on chromosome 3 of tomato. J. Amer. Soc. Hort. Sci. 134:281-288.
Miller, J.C. and S.D. Tanksley. 1990. RJLP analysis of phylogenetic relationships and genetic variation in the genus Lycopersicon. Theor. Appl. Genet. 80:437-448.

Mutchler, M.A., S.D. Tanksley, and C.M. Rick. 1987. 1987 linkage maps of the tomato (Lycopersicon esculentum). Rept. Tomato Genet. Coop. 37:5-34.

Peace, C.P. and J. Norelli. 2009. Genomics approaches to crop improvement in Rosaceae, p. 19-53. In: Folta, K. and S. Gardiner (eds.). Genetics and genomics of Rosaceae. Springer, New York, NY.

Scott, J.W. 2002. A breeder's perspective on the use of molecular techniques for improving fruit quality. HortScience 37:464-467.

Stall, R.E. 1991. Graywall, p. 65. In: In: Jones, J.B., R.E. Stall, and T.A. Zitter (eds.). Compendium of tomato diseases. APS Press, St Paul, MN

Suzuki, A., K. Suwabe, and K. Yano. 2009. Web databases of omics data in tomato. J. Japan Hort. Sci. 78:23-31.

Williams, C.E. and D.A. St. Clair. 1993. Phenetic relationships and levels of variability detected by restriction fragment length polymorphism and random amplified polymorphic DNA analysis of cultivated and wild accessions of $L y c O-$ persicon esculentum. Genome 36:619-630. 